Advances in Dynamical Systems and Applications (ADSA).

ISSN 0973-5321, Volume 16, Number 1, (2021) pp. 369-403

(C) Research India Publications

https://dx.doi.org/10.37622/ADSA/16.1.2021.369-403

\title{
Combined Drug Interventions and its Efficacy in the Reduction of COVID-19 Burden : A Within-Host Modeling Study with reference to HCQ and BCG Vaccination
}

\author{
Bishal Chhetri $^{\mathrm{a}}$, D K K Vamsi *a, Bhanu Prakash ${ }^{\mathrm{a}}$, and Carani B Sanjeevi ${ }^{\mathrm{b}, \mathrm{c}}$ \\ ${ }^{a}$ Department of Mathematics and Computer Science, Sri Sathya Sai Institute of Higher \\ Learning - SSSIHL, India. \\ ${ }^{\mathrm{b}}$ Vice-Chancellor, Sri Sathya Sai Institute of Higher Learning - SSSIHL, India. \\ ${ }^{\mathrm{c}}$ Department of Medicine, Karolinska Institute, Stockholm, Sweden.
}

\begin{abstract}
The COVID-19 pandemic has resulted in more 176 million cases and around 3.82 million deaths worldwide. Different drug interventions acting at multiple stages of the pathogenesis of COVID-19 can substantially reduce infectioninduced mortality. The current within-host mathematical modeling studies deals with the optimal combined drug intervention strategy and its efficacy in reducing the burden of COVID-19. The drug interventions considered here include Hydroxychloroquine (HCQ), the first BCG vaccine dose, and a booster dose of BCG administered at a later stage. In this work, we consider two scenarios involving the administration of these interventions. The findings of these studies include the following: the average infected cell count and viral load decreased the most when both the HCQ and BCG interventions were applied together in both scenarios. On the other hand, the average susceptible cell count decreased the best when HCQ alone was administered in both these scenarios. From the comparative effectiveness study it was observed that the basic reproduction number and viral count decreased the best when HCQ and BCG booster interventions were applied together, reinstating the fact obtained earlier in the optimal control setting. These findings may help physicians with decision making in the treatment of life-threatening COVID-19 pneumonia. This study involving different drug interventions is first of its kind.
\end{abstract}

Keywords COVID-19; Inflammatory Mediators; BCG Vaccine; HCQ; Optimal Control Problem;

${ }^{*}$ Corresponding Author 


\section{INTRODUCTION}

The humanity at this point of time is facing one the worst crisis in the last century in the form of pandemic COVID-19 caused by the Severe Acute Respiratory Syndrome Coronavirus-2 (SARS-CoV-2), which engulfed around 176 million cases and around 3.82 million deaths worldwide till date and this scenario continues to worsen [6]. Doctors, scientists, leaders of the states and many other professionals are working round the clock to root out the virus from our society. At this juncture mathematical modeling can play a crucial role in this journey as they are helpful in multi-fold. Firstly, they help in understanding the dynamics of infection and its spread in the society. They help in studying the success of various control measures that can be implemented in order to avoid the further damage. Secondly, the within-host mathematical modeling helps to study the dynamics of virus in the human body and can help us in understanding efficacy of different drug intervention acting at multiple stages of pathogenesis which in turn can help in identification of potential vaccine candidates. Some works dealing with population level studies and within-host studies for diseases such as Dengue, HIV, Influenza include $[15,26]$ and references within. A SAIU compartmental mathematical model that explains the transmission dynamics of COVID-19 is developed in [36]. The role of some of the control policies such as treatment, quarantine, isolation, screening, etc. are also applied to control the spread of infectious diseases [13, 28, 7]. Recent works dealing with population level studies and within-host studies for COVID-19 can be found in $[20,22,25,29,37]$.

The Bacillus Calmette-Guerin (BCG) vaccine was first mass produced in 1924 for tuberculosis disease. The BCG tuberculosis vaccine boosts the production of immune cells. A study proves that century-old BCG vaccine used to eradicate tuberculosis does reduce the chance of death from COVID-19 [2, 4]. It is found out that the countries with a $10 \%$ greater prevalence of the BCG vaccine also had a $10.4 \%$ reduction in COVID19 mortality [16, 12]. Recent work [9] showed that mandated BCG vaccination can be effective in fight against COVID-19. In fact mandated BCG predicted flattened curves for the spread of COVID-19 [9]. Bacillus Calmette-Guérin (BCG) vaccination (typically given at birth and/or during childhood) offers a long-lasting protective effect not only against tuberculosis (the intended target of BCG), but also against various other infectious diseases. Recent suggestions abound that BCG could be an effective tool in fighting against COVID-19. However, existing cross-national analyses are hampered by methodological weaknesses. For the most part, no effort has been made to exclude potential effects of reporting biases [9]. The nonspecific effects (NSEs) of immune responses induced by BCG vaccination protect against other infections seem to be due to its immunological memory eliciting lymphocytes response and trained immunity. 
The protective effect on other viral infection in humans are believed to be mediated by heterologous lymphocyte activation and the initiation of innate immune memory may be applicable to SARS CoV2 [32]. Several studies underscored reduction in respiratory tract infections and risk of pneumonia upon BCG vaccination of elderly people [32]. A randomized study showed that BCG vaccination prior to influenza vaccination in healthy individuals resulted in a significantly higher antibody response against influenza A (H1N1) compared to placebo [24].

The effectiveness of BCG in the promotion of long-lasting $\mathrm{T}$ cell immunity to human respiratory syncytial virus (hRSV) antigens was experimentally demonstrated without any observable adverse effects [32]. In a situation where an efficient vaccine for COVID-19 is unavailable BCG could be one of the alternative to reduce the disease burden.

Also, the anti-malarial drug Hydroxychloroquine (HCQ) has demonstrated in-vitro activity against SARS-CoV-2 [3]. HCQ along with other drugs like Azithromycin was administered initially to COVID-19 patients. Later medical guidelines were modified to administer HCQ alone to treat COVID-19 patients at the early course of the disease [3]. HCQ acts by preventing the SARS-CoV-2 virus from binding to cell membranes and also CQ/HCQ could inhibit viral entry by acting as inhibitors of the biosynthesis of sialic acids, critical actors of virus-cell ligand recognition [35].

\section{Pathogenesis of COVID-19}

Human to human transmission of SARS-CoV-2 occurs either through droplet infection or direct contact from an infected person. Transmission from asymptomatics and through faeco-oral route are also reported. After the entry of virus through droplet infection or through contact transmission from hands to the mucous membrane of mouth, nose or eyes. It is reported that Angiotensin Converting Enzyme-2 (ACE-2) plays crucial role in providing binding site to the viral structural protein(Spike proteinS) on the cell surface and subsequent entry into the host cell [42]. The SARS-CoV2 have much higher affinity towards ACE-2 receptors as compared with its previous conterparts(SARS-CoV and MERS-CoV). The ACE-2 expresses in lung alveolar Type-2 cells(AT2), liver cholangiocytes, colon colonocytes, esophagus, keratinocytes, endothelial cells of ileum, rectum and stomach, and proximal tubules of kidneys. AT2 secret surfactant, which reduces surface tension preventing collapsing of the alveoli and playing crucial role in oxygen diffusion across lungs and blood vessels. Viral antigens are presented by antigen presenting cells(APC) which are Human Leucocyte Antigen(HLA) cytotoxic T cell(also known as T-killer cell, cytotoxic T-lymphocyte, CD8+) [26]. The virus enters the cell with fusion of its membranes with host cell and begins transcription with ssRNA acting as template. Synthesis of the viral proteins 
takes place in the cytoplasm of the pneumocytes, new virus is released by budding and ready to infect new cell which is confirmed by presence of abundent viral antigens in the cytoplasm of the pneumocytes(AT2) in case of SARS-CoV [43]. Viremia (viral particle in the blood/serum) was also noticed by some authors along with very high levels of IL-6 especially among severe cases of Covid-19 illness leading to increase vascular permeability and impairment of organs [10]. Acute Respiratory Distress Syndrome(ARDS) is the common immunopathological event for all the aforesaid corona viral diseases. One of the main mechanisms in causation of ARDS is cytokine storm, deadly uncontrolled systemic inflammatory response due to the release of large quantity of pro-inflammatory cytokines and chemokines by immune effector cells. These cytokines are identified as IFN- $\alpha$, IFN- $\gamma$, IL-1b, IL-6, IL-2, IL-18, IL-33, TNF$\alpha$, TGF- $\beta$ and chemokines as CCL-2, CCL-3, CCL-5, CXCL-8, CXCL-9, CXCL-10. Severe infections correlated high levels of IL-6, IFN $\alpha$, CCL-5, CXCL-8 and CXCL$10[10,26]$. Also the viral load was noted to be crucial in determining severity of the disease and strongly correlated with the lung injury Murray score [29]. The cytokine storm is a violent attack by the immune system causing ARDS, multi-organ failure and eventually death [26].

From the above discussed pathogenesis it can be understood that the study of both the epithelial (pneumocytes) cells (including both the healthy and infected) and virus population levels and their changes over the time due to inflammatory mediators play a crucial in understanding the dynamics of pneumonia inflated COVID-19. Also as in case of HIV infection an optimal combined strategy with HCQ add BCG as controls may be a viable option for medical treatment in the context of COVID-19.

A detailed within host study involving crucial inflammatory mediators and the host immune response has been developed and discussed at length by the authors in [11]. The model in this study incorporated the roles of each of the pro-inflammatory cytokines and chemokine such as IFN- $\alpha$, IL-6, TNF- $\alpha$, and chemokines CCL-5, CXCL-8, CXCL10 released by the body. This work dealt with the natural history and course of infection of COVID-19. The authors also briefly discussed about the optimality and effectiveness of combined therapy involving one or more antiviral and one or more immuno-modulating drugs when administered together.

Motivated by the above discussions in the present work we study optimal drug intervention strategy and the efficacy of combined therapy in reducing the burden of COVID-19. As in earlier work [11] the model in this study incorporates the roles of each of the pro-inflammatory cytokines and chemokine such as IFN- $\alpha$, IL-6, TNF- $\alpha$, and chemokines CCL-5, CXCL-8, CXCL-10 released by the body. We additionally consider the role of different drug interventions. The drug interventions considered include 
HCQ, BCG first dose and BCG booster dose. We consider two scenarios involving administration of these interventions. In the first scenario we study the efficacy of drug interventions such as HCQ and BCG first dose administered at the present time. Initially we study their efficacy when administered individually and later we study the efficacy of the combined therapy involving administration of these two interventions HCQ, BCG first dose simultaneously at present. In the second scenario we assume that the BCG primary dose has been administered at birth to the individual and we study the efficacy of the drug interventions HCQ and BCG booster dose administered either individually or in combination at the present time. In both these scenarios the optimal intervention strategy is proposed based on two methods. In the first method these medical interventions are modeled as control interventions and a corresponding objective function and optimal control problem is formulated and discussed. Later using the comparative effectiveness method the optimal drug intervention strategy is proposed based on basic reproduction number and viral count. This study involving different drug interventions is first of its kind.

\section{OBJECTIVES OF THE PROPOSED STUDY}

1. To investigate the role of medical interventions such as HCQ and BCG vaccine by incorporating them as controls at specific sites in the pathogenesis.

2. To study and compare the dynamics of susceptible, infected cells and viral load with and without these control interventions.

3. To propose the optimal drug strategy in scenarios involving administration of single drug intervention, two drug interventions based on the average susceptible, infected cell count and average viral load.

4. To propose the optimal drug strategy using the comparative effectiveness studies.

\section{METHODS}

A within-host mathematical model incorporating drug interventions was studied as an optimal control problem. The Filippov Existence Theorem was used to obtain an optimal solution [27]. Characterization of optimal controls was done using Maximum Principle [27]. Numerical simulations involving with and without control interventions were performed for obtaining optimal drug regimen. Comparative effectiveness method [18] was used to study the change in basic reproduction number and virus count for single and multiple interventions. 


\section{MODEL WITH HCQ AND BCG FIRST DOSE}

Based on the pathogenesis of COVID-19 discussed above we now frame an optimal control problem to study the role of HCQ and BCG first dose in reducing the infected and viral load populations. We develop three compartment SIV model to study the dynamics of suceptible type II pneumocytes, infected type II pneumocytes and the virus population with drugs HCQ and BCG first dose as control measures. Since BCG vaccine is found to boost the immune response of the body [32], some fraction of the infected cells and the virus particles are removed because of the activation of the immune response. These decrease in the infected cell and viral load using BCG first dose is denoted by the control variable $\mu_{2 b c g f d}$ and $\mu_{3 b c g f d}$. Also since HCQ acts by preventing the SARS-CoV-2 virus from binding to cell membranes there is less chances of the cells coming in contact with the virus. Therefore, there is decrease in the cells that are suceptible to infection [35]. The control variable $\mu_{1 h c q}$ denotes the rate of decrease in the suceptible cells due to the use of HCQ drug. Here the control variables are assumed to be continuous and bounded. The dynamic model based on the above discussions with HCQ and BCG fist dose as control variables is described by the following system of nonlinear differential equations:

$$
\begin{aligned}
\frac{d S}{d t}= & \omega-\beta S V-\mu_{1 h c q}(t) S-\mu S \\
\frac{d I}{d t}= & \beta S V-\left(d_{1}+d_{2}+d_{3}+d_{4}+d_{5}+d_{6}\right) I \\
& -\left(\mu_{2 h c q}(t)+\mu_{2 b c g f d}(t)\right) I-\mu I \\
\frac{d V}{d t}= & \alpha I-\left(b_{1}+b_{2}+b_{3}+b_{4}+b_{5}+b_{6}\right) V \\
& -\mu_{1} V-\left(\mu_{3 h c q}(t)+\mu_{3 b c g f d}(t)\right) V
\end{aligned}
$$


Table 1

\begin{tabular}{|l|l|}
\hline \hline Symbols & Biological Meaning \\
\hline \hline$S$ & Healthy Type II Pneumocytes \\
\hline \hline$I$ & Infected Type II Pneumocytes \\
\hline \hline$\omega$ & Natural birth rate of Type II Pneumocytes \\
\hline \hline$V$ & Viral load \\
\hline \hline$\alpha$ & Rate at which healthy Pneumocytes are infected \\
\hline \hline$\mu$ & Burst rate of virus particles \\
\hline \hline$\mu_{1}$ & Natural death rate of Type II Pneumocytes \\
\hline \hline$d_{1}, \quad d_{2}, \quad d_{3}, \quad d_{4}, \quad d_{5}, \quad d_{6}$ & $\begin{array}{l}\text { Rates at which Infected Pneumocytes are removed because } \\
\text { of the release of cytokines and chemokines IL-6 } \\
\text { TNF- } \alpha, \quad \text { INF, CCL5, CXCL8, CXCL10 respectively }\end{array}$ \\
\hline \hline$b_{1}, \quad b_{2}, \quad b_{3}, \quad b_{4}, \quad b_{5}, \quad b_{6}$ & $\begin{array}{l}\text { Rates at which Virus is removed because of } \\
\text { the release of cytokines and chemokines IL-6 } \\
\text { TNF- } \alpha, \quad \text { INF, CCL5, CXCL8, CXCL10 respectively }\end{array}$ \\
\hline \hline$\mu_{1 h c q}, \quad \mu_{2 h c q}, \quad \mu_{3 h c q}$ & $\begin{array}{l}\text { Rates at which susceptible cells, } \\
\text { infected cells and viral load are reduced } \\
\text { due to HCQ drug. }\end{array}$ \\
\hline \hline$\mu_{2 b c g f d}, \quad \mu_{3 b c g f d}$ & $\begin{array}{l}\text { Rates at which infected cells and the } \\
\text { viral load are reduced due to BCG first dose }\end{array}$ \\
\hline \hline
\end{tabular}

System (2.1)-(2.3) could also be worked with delays. Delays can be introduced both in the state variables and control variables. But with delays complexity of the problem increases and to avoid these in this work we do not consider delay in the system (2.1)-(2.3). Some of the works where delays are considered in the models are; a fractional-order delayed malware propagation model is developed and studied in [41]. The impact of two different delays and the fractional order on the stability and the emergence of Hopf bifurcation of involved model is presented in [40] by developing a new fractional-order chemotherapy model with two different delays. Oscillations for a delayed predator-prey model with Hassell-Varley-type functional response and periodic property and asymptotic behavior for a discrete ratio-dependent food-chain system with delay can be found in ([39],[38]). The optimal control strategy of an SIR epidemic model with time delay in state and control variables is investigated in [31].

POSITIVITY AND BOUNDEDNESS We now establish the positivity and boundedness of the system (2.1)-(2.3) without considering the controls. 
Positivity: We now show that if the initial conditions of the system (2.1) - (2.3) are positive, then the solution remain positive for any future time. Using the equations (2.1)-(2.3), we get,

$$
\begin{array}{ll}
\left.\frac{d S}{d t}\right|_{S=0}=\omega \geq 0, & \left.\frac{d I}{d t}\right|_{I=0}=\beta S V \geq 0 \\
\left.\frac{d V}{d t}\right|_{V=0}=\alpha I \geq 0 . &
\end{array}
$$

Thus all the above rates are non-negative on the bounding planes (given by $S=0$, $I=0$, and $V=0$ ) of the non-negative region of the real space. So, if a solution begins in the interior of this region, it will remain inside it throughout time $t$. This happens because the direction of the vector field is always in the inward direction on the bounding planes as indicated by the above inequalities. Hence, we conclude that all the solutions of the the system $(2.1-2.3)$ remain positive for any time $t>0$ provided that the initial conditions are positive. This establishes the positivity of the solutions of the system (2.1-2.3). Next we will show that the solution is bounded .

\section{Boundedness:}

Here we will show that the solutions of the system (2.1)-(2.3) without the control variables is bounded. Let $N(t)=S(t)+I(t)+V(t)$

Let $x=d_{1}+d_{2}+d_{3}+d_{4}+d_{5}+d_{6}$ and $y=b_{1}+b_{2}+b_{3}+b_{4}+b_{5}+b_{6}$

Now,

$$
\begin{aligned}
\frac{d N}{d t} & =\frac{d S}{d t}+\frac{d I}{d t}+\frac{d V}{d t} \\
& =\omega-\mu(S+I)-\mu_{1} V-(x-\alpha) I-y V \\
& \leq \omega-\mu(S+I+V)
\end{aligned}
$$

with the assumption that $x>\alpha$ and $\mu=\mu_{1}$.

Here the integrating factor is $e^{\mu t}$. Therefore after integration we get, $N(t) \leq \frac{\omega}{\mu}+c e^{-\mu t}$. Now as $t \rightarrow \infty$ we get,

$$
N(t) \leq \frac{\omega}{\mu}
$$

Thus we have shown that the system (2.1-2.3) without control variable is positive and bounded. The solutions remains bounded for each bounded control variables. Therefore the biologically feasible region is given by the following set,

$$
\Omega=\left\{(S(t), I(t), V(t)) \in \mathbb{R}_{+}^{3}: S(t)+I(t)+V(t) \leq \frac{\omega}{\mu}, t \geq 0\right\}
$$


Definition of the objective function Let $U_{1}=\left(\mu_{1 h c q}, \mu_{2 h c q}, \mu_{3 h c q}\right), U_{2}=$ $\left(\mu_{2 b c g f d}, \mu_{3 b c g f d}\right)$

$$
\begin{aligned}
& J\left(U_{1}, U_{2}\right)= \\
& \int_{0}^{T}\left(A_{1}\left(\mu_{1 h c q}^{2}(t)+\mu_{2 h c q}^{2}(t)+\mu_{3 h c q}^{2}(t)\right)+A_{2}\left(\mu_{2 b c g f d}^{2}(t)+\mu_{3 b c g f d}^{2}(t)\right)-I(t)-V(t)\right) d t
\end{aligned}
$$

Here the cost function (2.4) represents the benefits of each of the interventions and the number of infected cells and viral load throughout the observation period. Our goal is to maximize the benefits of the implementation of each of the drug interventions and minimize the infected cells and viral load.

To reduce the complexity of the problem here we choose to model the control efforts via a linear combination of the quadratic terms. Also when the objective function is quadratic with respect to the control, differential equations arising from optimization have a known solution. Other functional forms sometimes lead to systems of differential equations that are difficult to solve [14], [23]. The coefficients $A_{i}$, for $i=1,2$ are the constants related to the overall benefits of each of the interventions.

The integrand of the cost function (2.4), denoted by

$L\left(I, V, U_{1}, U_{2}\right)=\left(A_{1}\left(\mu_{1 h c q}^{2}(t)+\mu_{2 h c q}^{2}(t)+\mu_{3 h c q}^{2}(t)\right)+A_{2}\left(\mu_{2 b c g f d}^{2}(t)+\mu_{3 b c g f d}^{2}(t)\right)-I(t)-V(t)\right)$

is called the Lagrangian of the running cost.

The admissible solution set for the optimal control problem (2.1) - (2.3) is given by,

$$
\Omega=\left\{\left(S, I, V, U_{1}, U_{2}\right) \mid S, I \text { and } V \text { that satisfy }(2.1)-(2.3) \forall U_{i} \in U\right\}
$$

where $U$ is the set of all admissible controls given by

$$
\begin{aligned}
& U=\left\{U_{1}=\left(\mu_{1 h c q}(t), \mu_{2 h c q}(t), \mu_{3 h c q}(t)\right), U_{2}=\left(\mu_{2 b c g f d}(t), \mu_{3 b c g f d}(t)\right)\right\}: \\
& \left.\left.U_{1}(t)\right) \in\left[0, U_{1} \max \right], U_{2}(t) \in\left[0, U_{2} \max \right], t \in[0, T]\right\}
\end{aligned}
$$

\subsection{Existence of Optimal Controls}

Now we will show that there exists optimal control solutions that maximizes the cost function (2.4). In order to prove the existence of optimal control function that maximize 
the cost functions within a finite time span $[0, T]$, we will show that the conditions stated in Theorem 4.1 of [17] is satisfied.

\section{Theorem 2.1.}

There exists a 5-tuple of optimal controls $\left(\mu_{1 h c q}^{*}(t), \mu_{2 h c q}^{*}(t), \mu_{3 h c q}^{*}(t), \mu_{2 b c g f d}^{*}(t)\right.$, $\left.\mu_{3 b c g f d}^{*}(t)\right)$ in the set of admissible controls $U$ such that the cost functional is maximized i.e.,

$J=$

$\left.\left.\left.\max _{\left(\mu_{1 h c q}, \mu_{2 h c q}, \mu_{3 h c q}, \mu_{2 b c g f d}, \mu_{3 b c g f d}\right) \in U}\left\{J\left[\mu_{1 h c q}(t), \mu_{2 h c q}(t), \mu_{3 h c q}(t)\right), \mu_{2 b c g f d}(t)\right), \mu_{3 b c g f d}(t)\right)\right]\right\}$ corresponding to the optimal control problem (2.1) - (2.3).

Proof. We will show that the following conditions are satisfied :

1. The solution set for the system (2.1) - (2.3) along with bounded controls must be non-empty, i.e., $\Omega \neq \phi$.

2. $\mathrm{U}$ is closed and convex and system should be expressed linearly in terms of the control variables with coefficients that are functions of time and state variables.

3. The Lagrangian $\mathrm{L}$ should be convex on $\mathrm{U}$ and $L\left(S, I, V, U_{1}, U_{2}\right) \geq$ $g\left(U_{1}, U_{2}\right)$, where $g$ is a continuous function of control variables such that $\left|\left(\mu_{1 h c q}, \mu_{2 h c q}, \mu_{3 h c q}, \mu_{2 b c g f d}, \mu_{3 b c g f d}\right)\right|^{-1} g\left(U_{1}, U_{2}\right)$

$\rightarrow \infty$ whenever $\left|\left(\mu_{1 h c q}, \mu_{2 h c q}, \mu_{3 h c q}, \mu_{2 b c g f d}, \mu_{3 b c g f d}\right)\right| \rightarrow \infty$, where $|$.$| is an$ $l^{2}(0, T)$ norm.

Now we will show that each of the conditions are satisfied :

1. From positivity and boundedness of solutions of the system (2.1) - (2.3) all solutions are bounded for each bounded control variable in $U$. Also, the right hand side of the system (2.1) - (2.3) satisfies Lipschitz condition with respect to state variables. Hence, using the positivity and boundedness condition and the existence of solution from Picard-Lindelof Theorem [30], we have satisfied condition 1.

2. $U$ is closed and convex by definition. Also, the system (2.1) - (2.3) is clearly linear with respect to controls such that coefficients are only state variables or functions dependent on time. Hence condition 2 is satisfied.

3. Choosing $g\left(\mu_{1 h c q}, \mu_{2 h c q}, \mu_{3 h c q}, \mu_{2 b c g f d}, \mu_{3 b b c g f d}\right)$

$=c\left(\mu_{1 h c q}^{2}+\mu_{2 h c q}^{2}+\mu_{3 h c q}^{2}+\mu_{2 b c g f d}^{2}+\mu_{3 b c g f d}^{2}\right)$ such that $c=\min \left\{A_{1}, A_{2}\right\}$, we can satisfy the condition 3 .

Hence there exists a control 5-tuple $\left(\mu_{1 h c q}, \mu_{2 h c q}, \mu_{3 h c q}, \mu_{2 b c g f d}, \mu_{3 b c g f d}\right) \in U$ that maximizes the cost function (2.4). 


\subsection{Characteriztion of Optimal Controls}

We now obtain the necessary conditions for optimal control functions using the Pontryagin's Maximum Principle [27] and also obtain the characteristics of the optimal controls.

The Hamiltonian for this problem is given by

$$
H\left(S, I, V, U_{1}, U_{2}, \lambda\right)=L\left(S, I, V, U_{1}, U_{2}\right)+\lambda_{1} \frac{\mathrm{d} S}{\mathrm{~d} t}+\lambda_{2} \frac{\mathrm{d} I}{\mathrm{~d} t}+\lambda_{3} \frac{\mathrm{d} V}{\mathrm{~d} t}
$$

Here $\lambda=\left(\lambda_{1}, \lambda_{2}, \lambda_{3}\right)$ is called co-state vector or adjoint vector.

Now the Canonical equations that relate the state variables to the co-state variables are given by

$$
\begin{aligned}
\frac{\mathrm{d} \lambda_{1}}{\mathrm{~d} t} & =-\frac{\partial H}{\partial S} \\
\frac{\mathrm{d} \lambda_{2}}{\mathrm{~d} t} & =-\frac{\partial H}{\partial I} \\
\frac{\mathrm{d} \lambda_{3}}{\mathrm{~d} t} & =-\frac{\partial H}{\partial V}
\end{aligned}
$$

Substituting the Hamiltonian value gives the canonical system

$$
\begin{aligned}
& \frac{\mathrm{d} \lambda_{1}}{\mathrm{~d} t}=\lambda_{1}\left(\beta V+\mu+\mu_{1 h c q}\right)-\lambda_{2} \beta V \\
& \frac{\mathrm{d} \lambda_{2}}{\mathrm{~d} t}=1+\lambda_{2}\left(x+\left(\mu_{2 h c q}+\mu_{2 b c g f d}+\mu\right)\right)-\lambda_{3} \alpha \\
& \frac{\mathrm{d} \lambda_{3}}{\mathrm{~d} t}=1+\lambda_{1} \beta S-\lambda_{2} \beta S+\lambda_{3}\left(y+\mu_{3 b c g f d}+\mu_{3 h c q}+\mu_{1}\right)
\end{aligned}
$$

where $x=d_{1}+d_{2}+d-3+d_{4}+d_{5}+d_{6}$ and $y=b_{1}+b_{2}+b_{3}+b_{4}+b_{5}+b_{6}$ along with transversality conditions $\lambda_{1}(T)=0, \lambda_{2}(T)=0, \lambda_{3}(T)=0$.

Now, to obtain the optimal controls, we will use the Hamiltonian minimization condition $\frac{\partial H}{\partial u_{i}}=0$, at $\mu^{*}$.

Differentiating the Hamiltonian and solving the equations, we obtain the optimal controls as 


$$
\begin{aligned}
\mu_{1 h c q}^{*} & =\min \left\{\max \left\{\frac{\lambda_{1} S}{2 A_{1}}, 0\right\}, \mu_{1 h c q} \max \right\} \\
\mu_{2 h c q}^{*} & =\min \left\{\max \left\{\frac{\lambda_{2} I}{2 A_{1}}, 0\right\}, \mu_{2 h c q} \max \right\} \\
\mu_{3 h c q}^{*} & =\min \left\{\max \left\{\frac{\lambda_{3} V}{2 A_{1}}, 0\right\}, \mu_{3 h c q} \max \right\} \\
\mu_{2 b c g f d}^{*} & =\min \left\{\max \left\{\frac{\lambda_{2} I}{2 A_{2}}, 0\right\}, \mu_{2 b c g f d} \max \right\} \\
\mu_{3 b c g f d}^{*} & =\min \left\{\max \left\{\frac{\lambda_{3} V}{2 A_{2}}, 0\right\}, \mu_{3 b c g f d} \max \right\}
\end{aligned}
$$

\section{OPTIMAL DRUG REGIMEN WITH HCQ AND BCG FIRST DOSE AS DRUG INTERVENTIONS}

In this section, we perform numerical simulations to understand the efficacy of single and two drug interventions and propose the optimal drug regimen in these scenarios. This is done by studying the effect of the corresponding controls on the dynamics of the system (2.1) - (2.3).

The efficacy of combinations of controls considered are:

1. Single drug intervention administration.

2. Two drug interventions administration.

For our simulations, we have taken the total number of days as $T=30$ and the parameter values from table 2 which are as in [11].

Table 2. Parameter Values

\begin{tabular}{|c|c|c|c|c|c|c|c|c|c|c|c|c|c|c|c|c|}
\hline$\omega$ & $\beta$ & $\mu$ & $\mu_{1}$ & $\alpha$ & $d_{1}$ & $d_{2}$ & $d_{3}$ & $d_{4}$ & $d_{5}$ & $d_{6}$ & $b_{1}$ & $b_{2}$ & $b_{3}$ & $b_{4}$ & $b_{5}$ & $b_{6}$ \\
\hline 10 & 0.05 & .1 & 1.1 & .5 & 0.027 & 0.22 & .1 & 0.428 & 0.01 & 0.01 & 0.1 & .1 & .08 & .11 & .1 & .07 \\
\hline
\end{tabular}

We first solve the state system numerically using Fourth Order Runge-Kutta method of order 4 in MATLAB. We take the initial values of state variables to be $S(0)=$ $3.2 \times 10^{5}, I(0)=0, V(0)=5$ and the initial values of the control parameters as zeros. Since the incubation period of SARS-COV-2 is approximately 4-5 days [11], initially we allow the system to grow for 5 days without considering any controls. The population of suceptible cells, infected cells and viral load after 5 days were calculated to be $3.1971 \times 10^{5}, 176,25$ respectively. Considering these as an initial values we 
simulate the system with controls and explore the individual roles of HCQ and BCG first dose and their combination therapy in reducing the infected cells and viral load. Now, to simulate the system with controls, we use the Forward-Backward Sweep method stating with the initial values of controls and solve the state system forward in time. Following this we solve the adjoint state system backward in time due to the transversality conditions, using the optimal state variables and initial values of optimal control.

Now, using the values of adjoint state variables, the values of optimal control are updated and with these updated control variables, we go through this process again. We continue this till the convergence criterion is met [27].

Since our aim is to maximize the objective function and the weight constants represent the benefits of each of the drugs used, the choice of values of the weight constant cannot be random. To assign the values for these weight constants we have used the concept of Hazard ratio of the drugs. In Survival analysis, Hazard Ratio (HR) plays a crucial role in determining the rate at which the people treated by drug may suffer a certain complication per unit time as the control population. Larger the hazard ratio, less is the benefit of using the drug. We use this concept in assigning weights to our objective function in our model. The hazard ratio of HCQ and BCG first dose are 0.34 and 0.21 respectively $[8,19]$. Therefore we assume that BCG first dose acts better or has more benefit in treating the infected patients compared to HCQ. We see that the hazard ratio of BCG first dose is $38 \%$ less than that of HCQ. Therefore the value of weight constant associated with HCQ is chosen $38 \%$ lesser than that of BCG.

Based on the above we choose, $A_{1}=124, A_{2}=200$. $A_{2}$ is chosen high compared to $A_{1}$ because it has the least hazard ratio.

In figure 1 the population of susceptible cells, infected cells and viral load are plotted without considering control variables. We see that the susceptible cells reduces and the infected cells increases exponentially due to the increase in viral load over a period of time. 

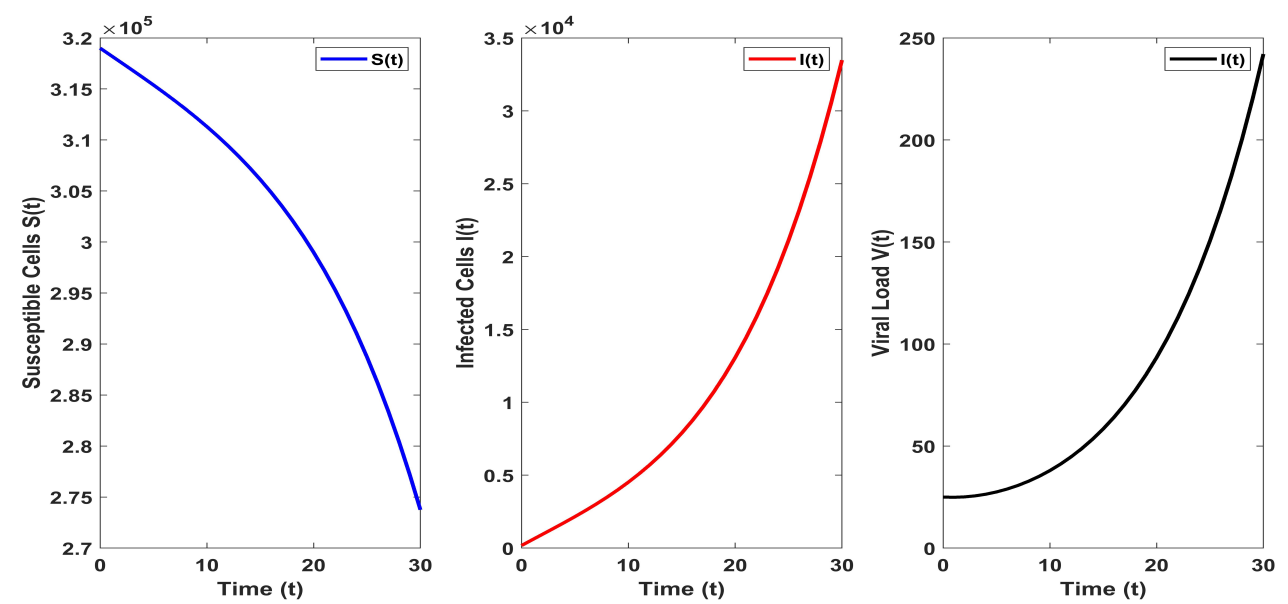

Figure 1. Figure depicting the $S, I, V$ populations for the system (2.1) - (2.3) without control interventions over the time. The exponential growth of the infected cells and viral load can be observed.

In figure $(2,3,4)$ we study the dynamics of susceptible, infected cells and viral load considering the individual and combined effect of HCQ and BCG first dose. We compare the curves of suceptible cells, infected cells and viral load with and without these controls and also calculate the average values of the susceptible cells, infected cells and the viral load with respect to each of the drug interventions HCQ and BCG first dose when administered individually and in combination. From figure 2 we see that the susceptible cells increases when BCG first dose is considered and decreases maximum when HCQ is considered. Therefore with HCQ as control the population of cells that can become infected is less. In figure $(3,4)$ the population of infected cells and viral load is plotted over 30 days. From these figures we observe that as control variables are considered the infected and viral load population starts decreasing. The average number of infected cells and viral load are lesser considering HCQ as control than BCG first dose. The use of HCQ could be a better option in reducing the infected cells and viral load population when controls are administered individually. But the infected cells and viral load population decreases the most when both the controls are administered together. These suggests that combined therapy could be a better option in minimizing the infected cells and viral load in the body than the individual drug therapy. 


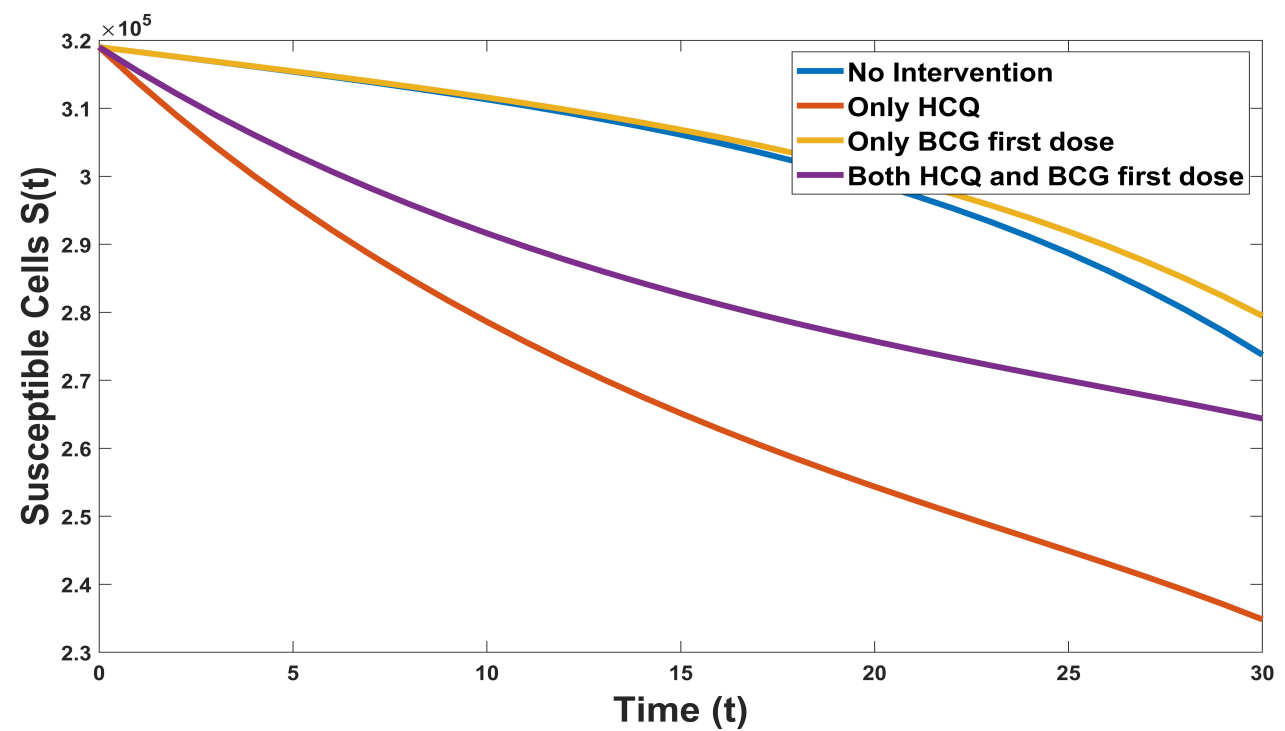

Figure 2. Figure depicting the dynamics of Susceptible cells $(S)$ under different combinations of the optimal controls $U_{1}^{*}, U_{2}^{*}$.

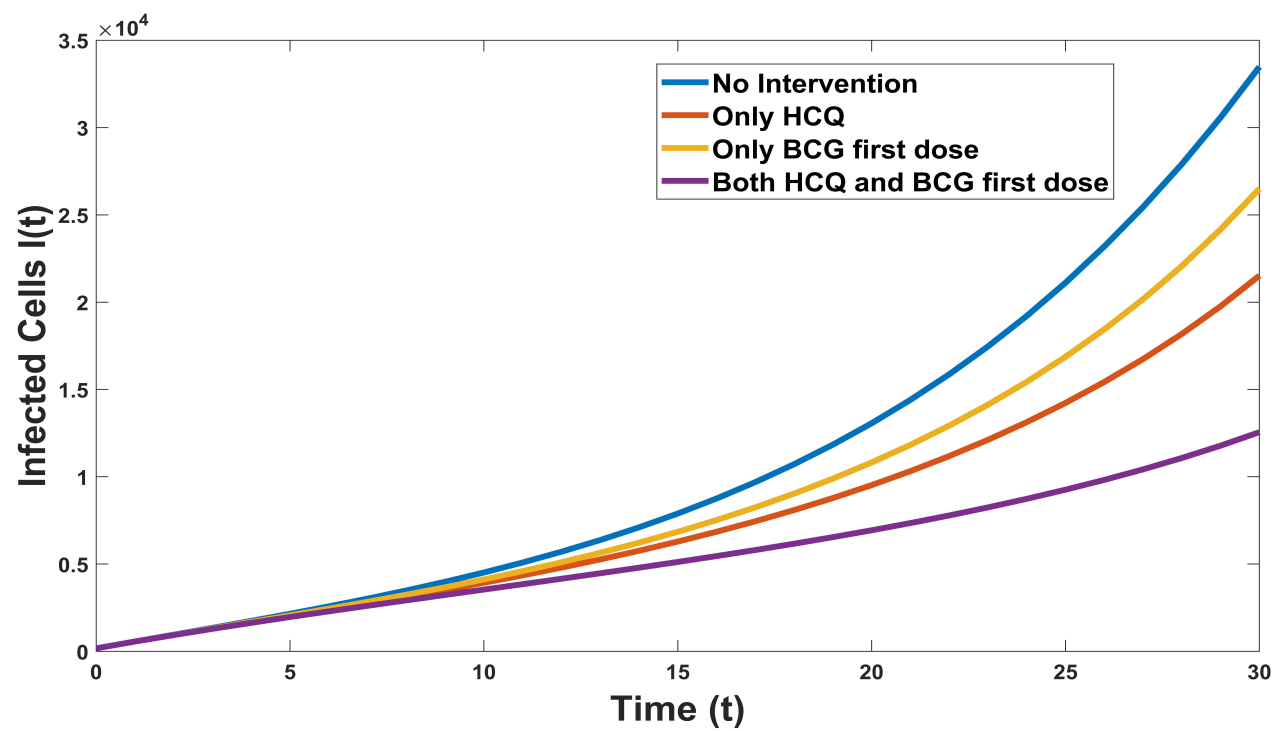

Figure 3. Figure depicting the dynamics of Infected cells $(I)$ under the different combinations of the optimal controls $U_{1}^{*}, U_{2}^{*}$. 


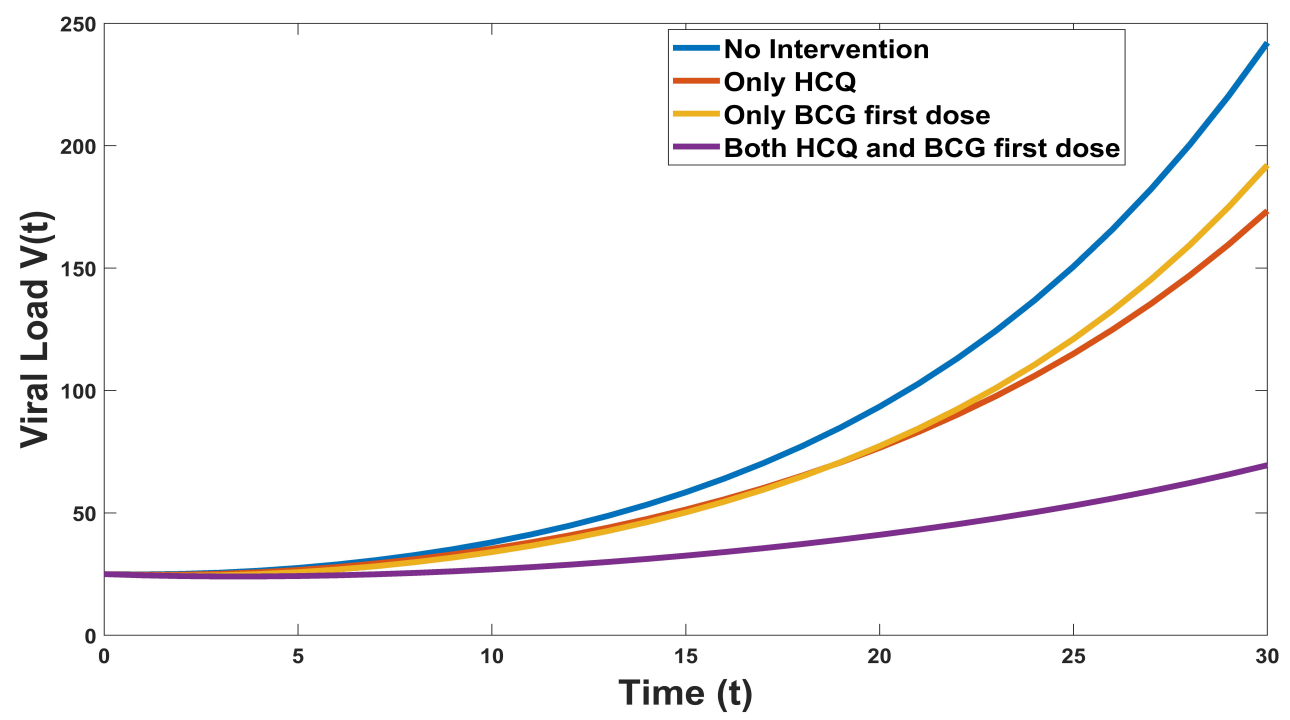

Figure 4. Figure depicting the dynamics of Viral load $(V)$ under different combinations of the optimal controls $U_{1}^{*}, U_{2}^{*}$.

In table 3 the average values of the susceptible cells, infected cells and the viral load with respect to each of the drug interventions HCQ, BCG first dose when administered individually and in combination is given. From this table 3 it can be seen that when both HCQ and BCG first dose are taken together the infected cells and viral load reduces the best followed by the drug HCQ $\left(U_{1}=U_{1}^{*}\right)$.

\begin{tabular}{|l|l|l|l|}
\hline Drug Combinations & Avg Suceptible cells & Avg Infected cells & Avg Viral load \\
\hline$U_{1}=U_{1}^{*}, U_{2}=U_{2}^{*}$ & $2.8587 \times 10^{5}$ & $5.5339 \times 10^{3}$ & 37.522 \\
\hline$U_{1}=U_{1}^{*}, U_{2}=0$ & $2.6936 \times 10^{5}$ & $7.814 \times 10^{3}$ & 67.452 \\
\hline$U_{1}=0, U_{2}=U_{2}^{*}$ & $3.042 \times 10^{5}$ & $9.018 \times 10^{3}$ & 69.541 \\
\hline \hline$U_{1}=0, U_{2}=0$ & $3.027 \times 10^{5}$ & $1.095 \times 10^{4}$ & 83.719 \\
\hline
\end{tabular}

Table 3. Table depicting the average values of the susceptible cells, infected cells and the viral load.

\section{MODEL WITH HCQ AND BCG BOOSTER DOSE}

In similar lines to section 2, in this section, we consider a control problem with the drug interventions HCQ and BCG booster dose as controls. The natural history for this model is in similar lines with the model presented by authors in [11]. We assume that the BCG primary dose is already administered to the patient at birth or in childhood at some point of time. Because of administration of BCG primary dose the individual will be now having an additional immunity component acquired due to BCG vaccine first 
dose. Incorporating this aspect the modified control problem is now given by

$$
\begin{aligned}
\frac{d S}{d t}= & \omega-\beta S V-\mu_{1 h c q}(t) S-\mu S \\
\frac{d I}{d t}= & \beta S V-\left(d_{1}+d_{2}+d_{3}+d_{4}+d_{5}+d_{6}\right) I \\
& -\left(\mu_{2 h c q}(t)+\mu_{2 b c g b d}(t)\right) I-\alpha_{1} I-\mu I \\
\frac{d V}{d t}= & \alpha I-\left(b_{1}+b_{2}+b_{3}+b_{4}+b_{5}+b_{6}\right) V \\
& -\alpha_{2} V-\mu_{1} V-\left(\mu_{3 h c q}(t)+\mu_{3 b c g b d}(t)\right) V
\end{aligned}
$$

Here the parameters $\alpha_{1}$ and $\alpha_{2}$ represent the rates of decrease of the infected and viral load because of the immunity gained due to BCG primary dose. Evidence from animal and human studies shows that there are significant differences in the immune response induced by different BCG vaccine strains [34]. Among the six widely used BCG vaccine strains, BCG Tice is the most efficacious BCG vaccine [21]. The values for $\alpha_{1}, \alpha_{2}$ will be based on the efficacy of the particular BCG vaccine strain. Using least square method discussed in [11] the values of $\alpha_{1}$ and $\alpha_{2}$ are estimated to be 0.1 and .01 . Also, the control interventions $\mu_{2 b c g b d}, \mu_{3 b c g b d}$ are the rates at which infected cells and viral load are reduced due to BCG booster dose.

\section{Definition of the objective function}

Let $U_{3}=\left(\mu_{1 h c q}, \mu_{2 h c q}, \mu_{3 h c q}\right), U_{4}=\left(\mu_{2 b c g b d}, \mu_{3 b c g b d}\right)$ $J\left(U_{3}, U_{4}\right)=\int_{0}^{T}\left(A_{3}\left(\mu_{1 h c q}^{2}(t)+\mu_{2 h c q}^{2}(t)+\mu_{3 h c q}^{2}(t)\right)+A_{4}\left(\mu_{2 b c g b d}^{2}(t)+\mu_{3 b c g b d}^{2}(t)\right)-I(t)-V(t)\right) d t$

The admissible solution set for the optimal control problem (4.1) - (4.3) is given by,

$$
\Omega=\left\{\left(S, I, V, U_{3}, U_{4}\right) \mid S, I \text { and } V \text { that satisfy }(4.1)-(4.4) \forall U_{i} \in U\right\}
$$

where $U$ is the set of all admissible controls given by

$$
\begin{aligned}
& U=\left\{U_{3}=\left(\mu_{1 h c q}(t), \mu_{2 h c q}(t), \mu_{3 h c q}(t)\right), U_{4}=\left(\mu_{2 b c g b d}(t), \mu_{3 b c g b d}(t)\right):\right. \\
& \left.\left.U_{3}(t)\right) \in\left[0, U_{1} \max \right], U_{4}(t) \in\left[0, U_{4} \max \right], t \in[0, T]\right\} .
\end{aligned}
$$


The existence of optimal controls and characterization of optimal controls for the model (4.1) - (4.4) are in similar lines to sections 2.1 and 2.2 and they are discussed in Appendix.

\section{NUMERICAL SIMULATION WITH HCQ AND BCG BOOSTER DOSE AS CONTROLS}

In this section, we perform numerical simulations to understand the efficacy of HCQ and BCG booster dose. This is done by studying the effect of the corresponding controls on the dynamics of the system (4.1) - (4.4). Here the parameter values and the initial conditions are considered same as in the previous case. Since we assume here that BCG first dose is already given and immune response is activated the role or the benefit of BCG booster may not be as good as BCG first dose. Therefore we choose the value of weight constant associated with BCG booster dose as $150\left(A_{4}=150\right)$, which is lesser that the value of weight constant associated with BCG first dose.

Similar to the first scenario here too we plot population of susceptible cells, infected cells and viral load with and without the controls. In figure $(5,6,7)$ we study the dynamics of susceptible, infected cells and viral load considering the individual and combined effect of HCQ and BCG booster dose. We compare the curves of suceptible cells, infected cells and viral load with and without these controls and also calculate the average values of the susceptible cells, infected cells and the viral load with respect to each of the drug interventions HCQ and BCG booster dose when administered individually and in combination. From figure 5 we see that the susceptible cells increases when BCG booster dose is considered and decreases maximum when HCQ is considered. Therefore with HCQ as control the population of cells that can become infected is less. In figure $(6,7)$ the population of infected cells and viral load is plotted over 30 days. From these figures we observe that as control variables are considered the infected and viral load population starts decreasing. The average number of infected cells and viral load are lesser considering HCQ as control than BCG booster dose. The use of HCQ could be a better option in reducing the infected cells and viral load population when controls are administered individually. But the infected cells and viral load population decreases the most when both the controls are administered together. These suggests that combined therapy could be a better option in minimizing the infected cells and viral load in the body than the individual drug therapy.

In the table 4 the average values of the susceptible cells, infected cells and the viral load with respect to each of these drug interventions when administered individually and in combination is listed. From this table 4 it can be seen that the combined therapy of drug HCQ $\left(U_{3}=U_{3}^{*}\right)$ and BCG booster dose $\left(U_{4}=U_{4}^{*}\right)$ reduces the infected cells and viral 
load the best followed by drug HCQ $\left(U_{3}=U_{3}^{*}\right)$.

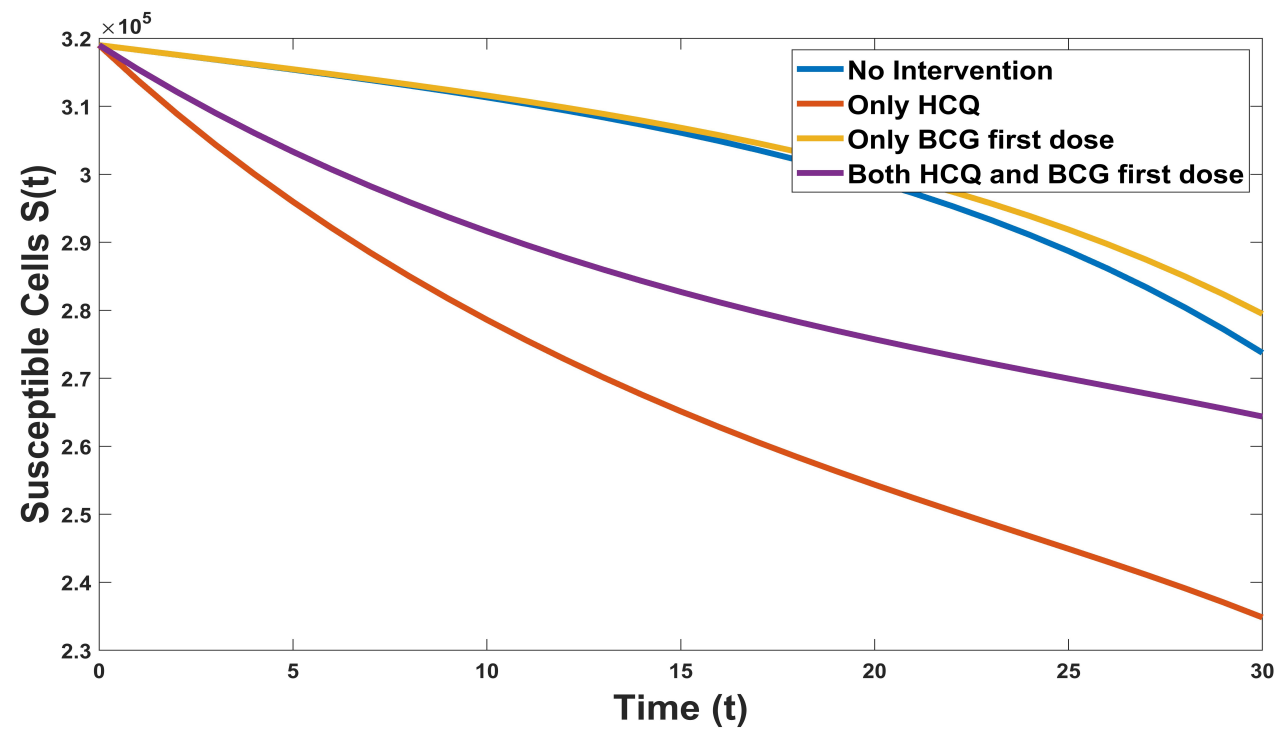

Figure 5. Figure depicting the dynamics of Susceptible cells $(S)$ under different combinations of the optimal controls $U_{3}^{*}, U_{4}^{*}$.

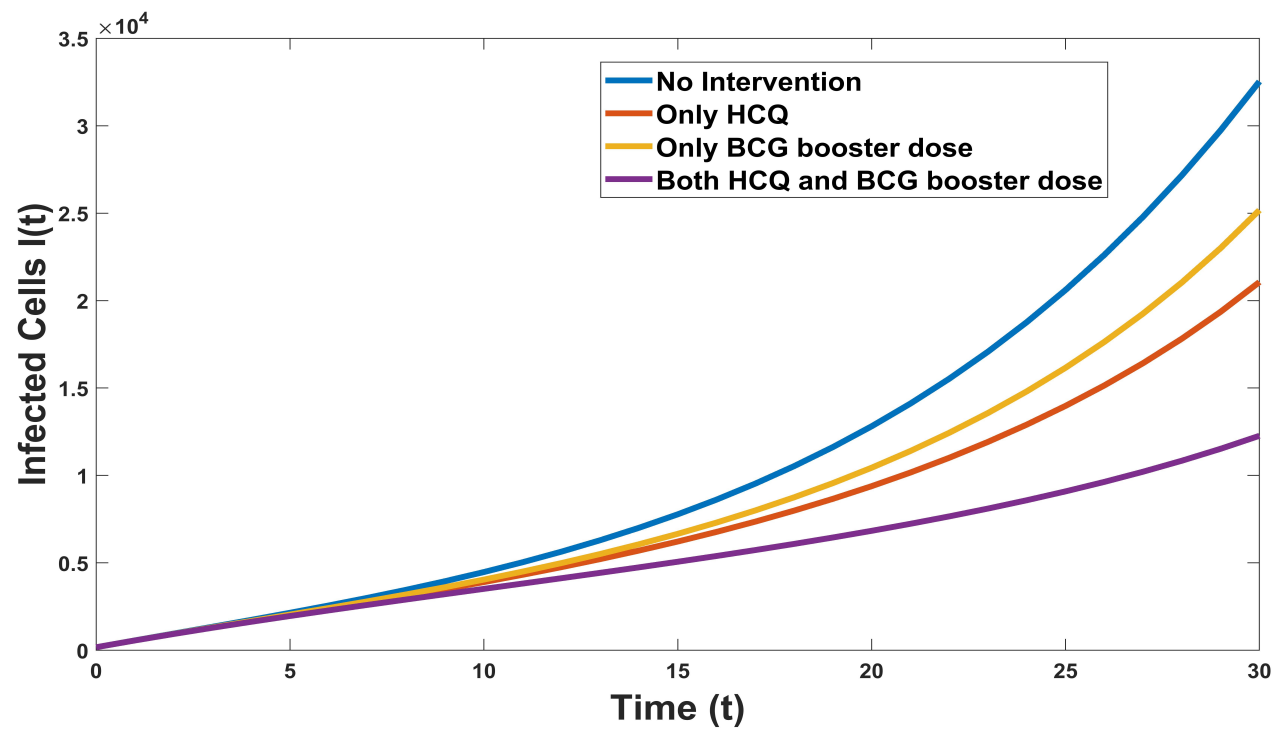

Figure 6. Figure depicting the dynamics of Infected cells $(I)$ under the different combinations of the optimal controls $U_{3}^{*}, U_{4}^{*}$. 


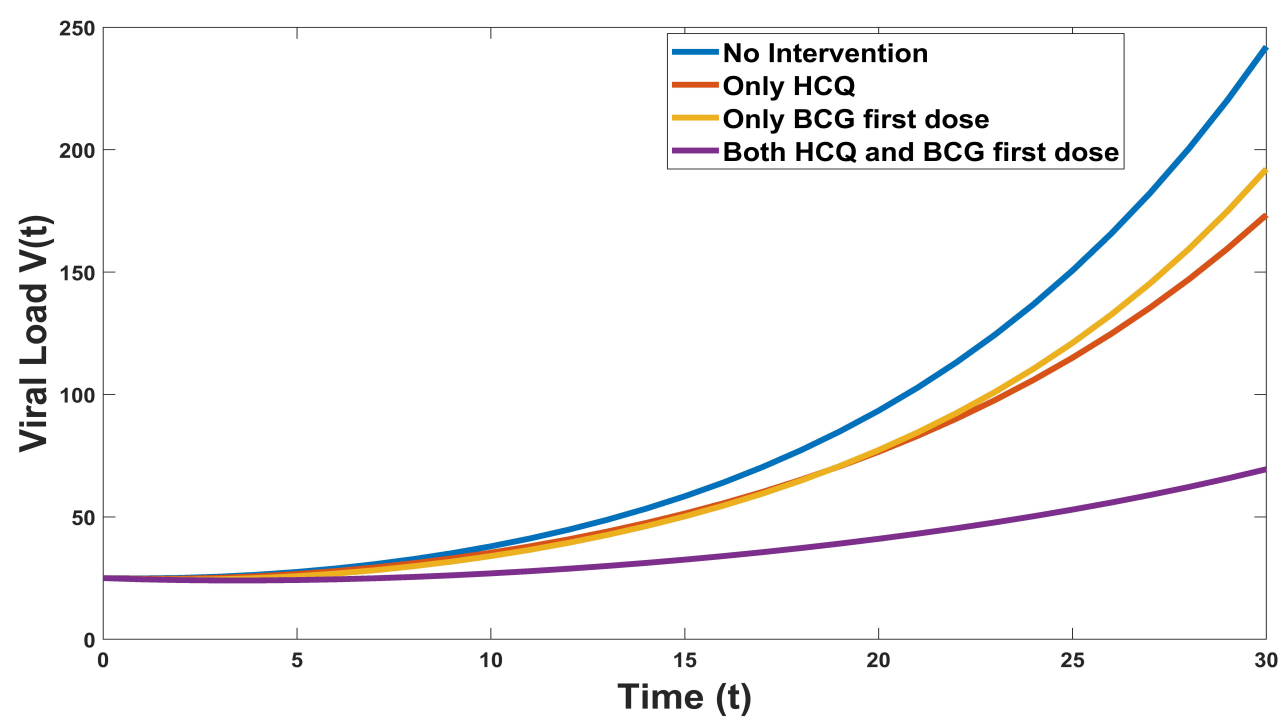

Figure 7. Figure depicting the dynamics of Viral load $(V)$ under different combinations of the optimal controls $U_{3}^{*}, U_{4}^{*}$.

\begin{tabular}{|l|l|l|l|}
\hline Drug Combinations & Avg Suceptible cells & Avg Infected cells & Avg Viral load \\
\hline$U_{3}=U_{3}^{*}, U_{4}=U_{4}^{*}$ & $2.8631 \times 10^{5}$ & $5.4484 \times 10^{3}$ & 36.9482 \\
\hline$U_{3}=U_{3}^{*}, U_{4}=0$ & $2.7 \times 10^{5}$ & $7.6933 \times 10^{3}$ & 66.32 \\
\hline$U_{3}=0, U_{4}=U_{4}^{*}$ & $3.0457 \times 10^{5}$ & $8.679 \times 10^{3}$ & 66.44 \\
\hline \hline$U_{3}=0, U_{4}=0$ & $3.0295 \times 10^{5}$ & $1.071 \times 10^{4}$ & 81.984 \\
\hline
\end{tabular}

Table 4. Table depicting the average values of the susceptible cells, infected cells and the viral load .

\section{COMPARATIVE EFFECTIVENESS STUDY}

In this section we do the comparative effectiveness study for the system

$$
\begin{aligned}
\frac{d S}{d t} & =\omega-\beta S V-\mu S \\
\frac{d I}{d t} & =\beta S V-\left(d_{1}+d_{2}+d_{3}+d_{4}+d_{5}+d_{6}\right) I-\mu I \\
\frac{d V}{d t} & =\alpha I-\left(b_{1}+b_{2}+b_{3}+b_{4}+b_{5}+b_{6}\right) V-\mu_{1} V
\end{aligned}
$$

The basic reproductive number for the system (6.1) - (6.3) as obtained in [1] is given by 


$$
\mathcal{R}_{0}=\frac{\beta \alpha \omega}{\mu\left(b_{1}+b_{2}+b_{3}+b_{4}+b_{5}+b_{6}+\mu_{1}\right)\left(d_{1}+d_{2}+d_{3}+d_{4}+d_{5}+d_{6}+\mu\right)}
$$

The disease-free equilibrium for the system can be seen to be

$$
E^{0}=\left(S^{0}, I^{0}, V^{0}\right)=\left(\frac{\omega}{\mu}, 0,0\right)
$$

and the endemic equilibrium to be

$$
\begin{aligned}
& \bar{S}=\frac{\left(b_{1}+b_{2}+b_{3}+b_{4}+b_{5}+b_{6}+\mu_{1}\right)\left(d_{1}+d_{2}+d_{3}+d_{4}+d_{5}+d_{6}+\mu\right)}{\alpha \beta} \\
& \bar{I}=\frac{\alpha \beta \omega-\mu\left(b_{1}+b_{2}+b_{3}+b_{4}+b_{5}+b_{6}+\mu_{1}\right)\left(d_{1}+d_{2}+d_{3}+d_{4}+d_{5}+d_{6}+\mu\right)}{\alpha \beta\left(d_{1}+d_{2}+d_{3}+d_{4}+d_{5}+d_{6}+\mu\right)} \\
& \bar{V}=\frac{\alpha \beta \omega-\mu\left(b_{1}+b_{2}+b_{3}+b_{4}+b_{5}+b_{6}+\mu_{1}\right)\left(d_{1}+d_{2}+d_{3}+d_{4}+d_{5}+d_{6}+\mu\right)}{\beta\left(b_{1}+b_{2}+b_{3}+b_{4}+b_{5}+b_{6}+\mu_{1}\right)\left(d_{1}+d_{2}+d_{3}+d_{4}+d_{5}+d_{6}+\mu\right)}
\end{aligned}
$$

Broadly we consider three kinds of interventions for this comparative effectiveness study.

1. Drugs that inhibit viral replication : Hydroxychloroquine(HCQ) does this job. So we now choose $\alpha$ to be $\alpha\left(1-\epsilon_{1}\right)$, where $\epsilon_{1}$ is the efficacy of HCQ.

2. Drugs that block virus binding to susceptible cells : Hydroxychloroquine(HCQ) does this job. So we now choose $\beta$ to be $\beta\left(1-\epsilon_{1}\right)$, where $\epsilon_{1}$ is the efficacy of HCQ.

3. Possible Immunotherapies : BCG vaccine does this job. So we now choose $\left(d_{1}+d_{2}+d_{3}+d_{4}+d_{5}+d_{6}\right)$ to be $\left(d_{1}+d_{2}+d_{3}+d_{4}+d_{5}+d_{6}\right)\left(1+\epsilon_{2}\right)$, where $\epsilon_{2}$ are the efficacy of BCG dosage.

$\mathcal{R}_{0}$ plays a crucial role in understanding the spread of infection in the individual and $\bar{V}$ determines the infectivity of virus in an individual. Taking the three kinds of interventions into consideration, we now have modified basic reproductive number $\mathcal{R}_{E}$ and modified virus count $\bar{V}_{E}$ of the endemic equilibrium to be

$$
\begin{aligned}
\mathcal{R}_{E} & =\frac{\alpha\left(1-\epsilon_{1}\right) \beta\left(1-\epsilon_{1}\right) \omega}{\mu\left(b_{1}+b_{2}+b_{3}+b_{4}+b_{5}+b_{6}+\mu_{1}\right)\left(\left(d_{1}+d_{2}+d_{3}+d_{4}+d_{5}+d_{6}\right)\left(1+\epsilon_{2}\right)+\mu\right)} \\
\overline{V_{E}} & =\frac{\alpha\left(1-\epsilon_{1}\right) \omega}{\left(b_{1}+b_{2}+b_{3}+b_{4}+b_{5}+b_{6}+\mu_{1}\right)\left(\left(d_{1}+d_{2}+d_{3}+d_{4}+d_{5}+d_{6}\right)\left(1+\epsilon_{2}\right)+\mu\right)} \\
& -\frac{\mu}{\beta\left(1-\epsilon_{1}\right)}
\end{aligned}
$$


The efficacy of these interventions was chosen based on the hazard ratios for each of these interventions. As the efficacy of each intervention ranges from 0 to 1 , the highest preference was given to the intervention with least hazard ratio. Since BCG first dose has the least hazard ratio among three interventions, we chose the efficacy of BCG first dose as $\epsilon_{2}=0.9$. As HCQ has the second highest hazard ratio among three interventions, we chose the efficacy of HCQ as $\epsilon_{1}=0.6$. The efficacy of BCG booster dose was chosen as $\epsilon_{2}=0.3$.

We now perform the comparative effectiveness study of these interventions by calculating the percentage reduction of $\mathcal{R}$, and $\bar{V}$ for single and multiple combination of these interventions. Percentage reduction of $\mathcal{R}_{0}$ and $\bar{V}$ are given by

Percentage reduction of $\mathcal{R}_{0}=\left[\frac{\mathcal{R}_{0}-\mathcal{R}_{E_{j}}}{\mathcal{R}_{0}}\right] \times 100$

Percentage reduction of $\bar{V}=\left[\frac{\bar{V}-\bar{V}_{E_{j}}}{\bar{V}}\right] \times 100$

where $\mathrm{j}$ stands for $\epsilon_{1}, \epsilon_{2}$ or combinations thereof.

Since we have 2 interventions, we consider $4\left(=2^{2}\right)$ different combinations of these drugs.

\begin{tabular}{|c|c|c|c|c|c|}
\hline No. & Intervention & $\begin{array}{c}\text { \%age change } \\
\text { in } \mathcal{R}_{0}\end{array}$ & Rank & $\begin{array}{c}\text { \%age change } \\
\text { in } \bar{V}\end{array}$ & Rank \\
\hline 1 & Nil & 0 & 1 & 0 & 1 \\
\hline & HCQ and BCG First Dose & & & & \\
\hline 2 & $\epsilon_{1}$ & 84 & 3 & 367.6 & 3 \\
\hline 3 & $\epsilon_{2}$ & 44.43 & 2 & 109.5 & 2 \\
\hline 4 & $\epsilon_{1} \epsilon_{2}$ & 91.11 & 4 & 411.4 & 4 \\
\hline & HCQ and BCG Booster Dose & & & & \\
\hline 5 & $\epsilon_{1}$ & 84 & 3 & 367.6 & 3 \\
\hline 6 & $\epsilon_{2}$ & 21.04 & 2 & 51.86 & 2 \\
\hline 7 & $\epsilon_{1} \epsilon_{2}$ & 87.37 & 4 & 388.34 & 4 \\
\hline
\end{tabular}

Table 5. Comparative Effectiveness Study

In the Table 5, the comparative effectiveness is calculated and measured on a scale from 1 to 4 with 1 denoting the lowest comparative effectiveness while 4 denoting the highest comparative effectiveness. The conclusions from this study are the following.

1. When single drug intervention is administered, HCQ outperforms BCG vaccination for either of the dosages w.r.t reduction in both $\mathcal{R}_{0}$ and $\bar{V}$ (refer rows 
$2,3,5,6$ in table 5).

2. When HCQ and BCG vaccine are both administered together, the best reduction in $\mathcal{R}_{0}$ and $\bar{V}$ is seen (refer rows 4,7 in table 5).

\section{DISCUSSIONS AND CONCLUSIONS}

In this work we have considered three drugs interventions namely, $\mathrm{HCQ}, \mathrm{BCG}$ first dose and BCG booster dose and studied their efficacy for treatment of COVID-19 when applied individually or in combination. This study is done in two ways. The first by modeling these interventions as control interventions and studying the optimal control problem. The second study was the comparative effectiveness study.

Conclusions from the optimal control studies and comparative effectiveness studies suggest the following.

1. All HCQ and BCG interventions when administered individually or in combination reduce the infected cells and viral load significantly.

2. The average infected cell count and viral load decreased the best when both the HCQ and BCG interventions were applied together.

3. The average susceptible cell count decreased the best when HCQ alone was administered.

4. From the comparative effectiveness study it was observed that the basic reproduction number and viral count decreased the best when HCQ and BCG booster interventions were applied together, reinstating the fact obtained earlier in the optimal control setting.

This study supports the findings in the recent work $[9,33,5]$ which state that

- "Mandated BCG vaccination can be effective in fight against COVID-19. Mandated BCG predicted flattened curves for the spread of COVID-19."

- "BCG vaccination might be associated with a decrease in the incidence of sickness during the COVID-19 pandemic."

- "A combination of hydroxychloroquine, vitamin and zinc tablets along with homeopathic medicines apart from separate medical facilities for policemen and women who contract COVID-19 has helped control the virus among the uniformed personnel." 


\section{AUTHORS CONTRIBUTIONS}

- First author : Performed research; contributed for theoretical analysis and numerical simulations; analyzed data

- Second/Corresponding author : Conceived the idea; contributed for theoretical analysis and numerical simulations; majorly wrote the entire manuscript

- Third author : Contributed for theoretical analysis; almost did the entire comparative effectiveness study

- Fourth/Senior author : Conceived the idea; contributed for theoretical analysis; gave intellectual inputs; proof read the entire manuscript

\section{DEDICATION}

The authors dedicate this paper to the founder chancellor of SSSIHL, Bhagawan Sri Sathya Sai Baba. The corresponding author also dedicates this paper to his loving elder brother D. A. C. Prakash who still lives in his heart and the first author also dedicates this paper to his loving father Purna Chhetri.

\section{BIBLIOGRAPHY}

[1] https://arxiv.org/abs/2005.02261.

[2] https://www.dailymail.co.uk/sciencetech/article-8509655/bcg-does-protectagainst-covid-19-study-confirms.html.

[3] https://www.ndtv.com/india-news/health-ministry-okays-anti-malarial-drug-hcqfor-early-course-of-covid-19-2245829.

[4] https://www.newscientist.com/article/2242866-bcg-vaccine-helps-fightinfections-by-boosting-immune-cell-production/.

[5] https://www.tribuneindia.com/news/nation/hcq-vitamin-tablets-help-mumbaicops-beat-covid-19-96751.

[6] https://www.worldometers.info/coronavirus/.

[7] M Soledad Aronna, Roberto Guglielmi, and Lucas M Moschen, A model for covid-19 with isolation, quarantine and testing as control measures, arXiv preprint arXiv:2005.07661 (2020). 
[8] Samia Arshad, Paul Kilgore, Zohra S Chaudhry, Gordon Jacobsen, Dee Dee Wang, Kylie Huitsing, Indira Brar, George J Alangaden, Mayur S Ramesh, John E McKinnon, et al., Treatment with hydroxychloroquine, azithromycin, and combination in patients hospitalized with covid-19, International journal of infectious diseases 97 (2020), 396-403.

[9] Martha K Berg, Qinggang Yu, Cristina E Salvador, Irene Melani, and Shinobu Kitayama, Mandated bacillus calmette-guérin (bcg) vaccination predicts flattened curves for the spread of covid-19, Medrxiv (2020).

[10] Xiaohua Chen, Binghong Zhao, Yueming Qu, Yurou Chen, Jie Xiong, Yong Feng, Dong Men, Qianchuan Huang, Ying Liu, Bo Yang, et al., Detectable serum sars-cov-2 viral load (rnaaemia) is closely associated with drastically elevated interleukin 6 (il-6) level in critically ill covid-19 patients, medRxiv (2020).

[11] Bishal Chhetri, Vijay M Bhagat, DKK Vamsi, VS Ananth, Bhanu Prakash, Roshan Mandale, Swapna Muthusamy, and Carani B Sanjeevi, Within-host mathematical modeling on crucial inflammatory mediators and drug interventions in covid19 identifies combination therapy to be most effective and optimal, Alexandria Engineering Journal (2020).

[12] Camila Covián, Angello Retamal-Díaz, Susan M Bueno, and Alexis M Kalergis, Could bcg vaccination induce protective trained immunity for sars-cov-2?, Frontiers in Immunology 11 (2020), 970.

[13] Ramses Djidjou-Demasse, Yannis Michalakis, Marc Choisy, Micea T Sofonea, and Samuel Alizon, Optimal covid-19 epidemic control until vaccine deployment, medRxiv (2020).

[14] _ Optimal covid-19 epidemic control until vaccine deployment, medRxiv (2020).

[15] Jeffrey W Eaton, Leigh F Johnson, Joshua A Salomon, Till Bärnighausen, Eran Bendavid, Anna Bershteyn, David E Bloom, Valentina Cambiano, Christophe Fraser, Jan AC Hontelez, et al., Hiv treatment as prevention: systematic comparison of mathematical models of the potential impact of antiretroviral therapy on hiv incidence in south africa, PLoS Med 9 (2012), no. 7, e1001245.

[16] Luis E Escobar, Alvaro Molina-Cruz, and Carolina Barillas-Mury, Bcg vaccine protection from severe coronavirus disease 2019 (covid-19), Proceedings of the National Academy of Sciences (2020). 
[17] Wendell H Fleming and Raymond W Rishel, Deterministic and stochastic optimal control, vol. 1, Springer Science \& Business Media, 2012.

[18] Winston Garira and Dephney Mathebula, Development and application of multiscale models of acute viral infections in intervention research, Mathematical Methods in the Applied Sciences 43 (2020), no. 6, 3280-3306.

[19] Evangelos J Giamarellos-Bourboulis, Maria Tsilika, Simone Moorlag, Nikolaos Antonakos, Antigone Kotsaki, Jorge Domínguez-Andrés, Evdoxia Kyriazopoulou, Theologia Gkavogianni, MariaEvangelia Adami, Georgia Damoraki, et al., Activate: randomized clinical trial of bcg vaccination against infection in the elderly, Cell 183 (2020), no. 2, 315323.

[20] Giulia Giordano, Franco Blanchini, Raffaele Bruno, Patrizio Colaneri, Alessandro Di Filippo, Angela Di Matteo, and Marta Colaneri, Modelling the covid-19 epidemic and implementation of population-wide interventions in italy, Nature Medicine (2020), 1-6.

[21] Marcus A Horwitz, Günter Harth, Barbara Jane Dillon, and Saša Masleša-Galić, Commonly administered bcg strains including an evolutionarily early strain and evolutionarily late strains of disparate genealogy induce comparable protective immunity against tuberculosis, Vaccine 27 (2009), no. 3, 441-445.

[22] Adam J Kucharski, Timothy W Russell, Charlie Diamond, Yang Liu, John Edmunds, Sebastian Funk, Rosalind M Eggo, Fiona Sun, Mark Jit, James D Munday, et al., Early dynamics of transmission and control of covid-19: a mathematical modelling study, The lancet infectious diseases (2020).

[23] Sunmi Lee, Gerardo Chowell, and Carlos Castillo-Chávez, Optimal control for pandemic influenza: the role of limited antiviral treatment and isolation, Journal of Theoretical Biology 265 (2010), no. 2, 136-150.

[24] Jenneke Leentjens, Matthijs Kox, Robin Stokman, Jelle Gerretsen, Dimitri A Diavatopoulos, Reinout van Crevel, Guus F Rimmelzwaan, Peter Pickkers, and Mihai G Netea, Bcg vaccination enhances the immunogenicity of subsequent influenza vaccination in healthy volunteers: a randomized, placebo-controlled pilot study, The Journal of infectious diseases 212 (2015), no. 12, 1930-1938.

[25] Chentong $\mathrm{Li}$, Jinhu $\mathrm{Xu}$, Jiawei Liu, and Yicang Zhou, The within-host viral kinetics of sars-cov-2, bioRxiv (2020). 
[26] Xiaowei Li, Manman Geng, Yizhao Peng, Liesu Meng, and Shemin Lu, Molecular immune pathogenesis and diagnosis of covid-19, Journal of Pharmaceutical Analysis (2020).

[27] Daniel Liberzon, Calculus of variations and optimal control theory: a concise introduction, Princeton University Press, 2011.

[28] Gustavo Barbosa Libotte, Fran Sérgio Lobato, Gustavo Mendes Platt, and Antônio J Silva Neto, Determination of an optimal control strategy for vaccine administration in covid-19 pandemic treatment, Computer methods and programs in biomedicine 196 (2020), 105664.

[29] Yingxia Liu, Yang Yang, Cong Zhang, Fengming Huang, Fuxiang Wang, Jing Yuan, Zhaoqin Wang, Jinxiu Li, Jianming Li, Cheng Feng, et al., Clinical and biochemical indexes from 2019-ncov infected patients linked to viral loads and lung injury, Science China Life Sciences 63 (2020), no. 3, 364-374.

[30] Evgeny Makarov and Bas Spitters, The picard algorithm for ordinary differential equations in coq, International Conference on Interactive Theorem Proving, Springer, 2013, pp. 463-468.

[31] E Mohamed, R Mostafa, and B Elhabib, Optimal control of an sir model with delay in state and control variables, ISRN Biomathematics (2013).

[32] Prasanta Raghab Mohapatra, Baijayantimala Mishra, and Bijayini Behera, Bcg vaccination induced protection from covid-19, Indian Journal of Tuberculosis (2020).

[33] Simone JCFM Moorlag, Rosanne C van Deuren, Cornelis H van Werkhoven, Martin Jaeger, Priya Debisarun, Esther Taks, Vera P Mourits, Valerie ACM Koeken, L Charlotte J de Bree, Thijs Ten Doesschate, et al., Safety and covid19 symptoms in individuals recently vaccinated with bcg: a retrospective cohort study, Cell Reports Medicine (2020), 100073.

[34] Nicole Ritz, Willem A Hanekom, Roy Robins-Browne, Warwick J Britton, and Nigel Curtis, Influence of bcg vaccine strain on the immune response and protection against tuberculosis, FEMS microbiology reviews 32 (2008), no. 5, 821-841.

[35] Eugenia Quiros Roldan, Giorgio Biasiotto, Paola Magro, and Isabella Zanella, The possible mechanisms of action of 4-aminoquinolines 
(chloroquine/hydroxychloroquine) against sars-cov-2 infection (covid-19): A role for iron homeostasis?, Pharmacological research (2020), 104904.

[36] Piu Samui, Jayanta Mondal, and Subhas Khajanchi, A mathematical model for covid-19 transmission dynamics with a case study of india, Chaos, Solitons \& Fractals 140 (2020), 110173.

[37] Esteban Abelardo Hernandez Vargas and Jorge X Velasco-Hernandez, In-host modelling of covid-19 kinetics in humans, medRxiv (2020).

[38] Changjin Xu and Peiluan Li, Oscillations for a delayed predator-prey model with hassell-varley-type functional response, Comptes rendus biologies 338 (2015), no. 4, 227-240.

[39] Changjin $\mathrm{Xu}$, Peiluan Li, and Maoxin Liao, Periodic property and asymptotic behavior for a discrete ratio-dependent food-chain system with delays, Discrete Dynamics in Nature and Society 2020 (2020).

[40] Changjin Xu, Peiluan Li, Maoxin Liao, and Shuai Yuan, Bifurcation analysis for a fractional-order chemotherapy model with two different delays, Mathematical Methods in the Applied Sciences 43 (2020), no. 3, 1053-1083.

[41] Changjin $\mathrm{Xu}$, Maoxin Liao, and Peiluan Li, Bifurcation of a fractional-order delayed malware propagation model in social networks, Discrete Dynamics in Nature and Society 2019 (2019).

[42] Chayu Yang and Jin Wang, A mathematical model for the novel coronavirus epidemic in wuhan, china, Mathematical Biosciences and Engineering 17 (2020), no. 3, 2708-2724.

[43] Sherif R Zaki and Cynthia S Goldsmith, Sars coronavirus infection: pathology and pathogenesis of an emerging virus disease, Coronaviruses with Special Emphasis on First Insights Concerning SARS, Springer, 2005, pp. 87-99. 


\section{APPENDIX}

\subsection{Existence of Optimal Controls}

\section{Theorem 8.1.}

There exists a 5-tuple of optimal controls $\left(\mu_{1 h c q}^{*}(t), \mu_{2 h c q}^{*}(t), \mu_{3 h c q}^{*}(t), \mu_{2 b c g b d}^{*}(t)\right.$, $\mu_{3 b c g b d}^{*}(t)$ in the set of admissible controls $U$ such that the cost functional is maximized i.e.,

$J=$

$\left.\left.\left.\max _{\left(\mu_{1 h c q}, \mu_{2 h c q}, \mu_{3 h c q}, \mu_{2 b c g b d}, \mu_{3 b c g b d}\right) \in U}\left\{J\left[\mu_{1 h c q}(t), \mu_{2 h c q}(t), \mu_{3 h c q}(t)\right), \mu_{2 b c g b d}(t)\right), \mu_{3 b c g b d}(t)\right)\right]\right\}$ corresponding to the optimal control problem (4.1) - (4.4).

Proof. In order to show the existence of optimal control functions, we will show that the following conditions are satisfied :

1. The solution set for the system (4.1) - (4.4) along with bounded controls must be non-empty, i.e., $\Omega \neq \phi$.

2. $U$ is closed and convex and system should be expressed linearly in terms of the control variables with coefficients that are functions of time and state variables.

3. The Lagrangian $\mathrm{L}$ should be convex on $\mathrm{U}$ and $L\left(S, I, V, U_{3} . U_{4}\right) \quad \geq$ $g\left(U_{3}, U_{4}\right)$, where $g$ is a continuous function of control variables such that $\left|\left(\mu_{1 h c q}, \mu_{2 h c q}, \mu_{3 h c q}, \mu_{2 b c g b d}, \mu_{3 b c g b d}\right)\right|^{-1}$

$g\left(\mu_{1 h c q}, \mu_{2 h c q}, \mu_{3 h c q}, \mu_{2 b c g b d}, \mu_{3 b c g b d}\right) \rightarrow \infty$

whenever $\left|\left(\mu_{1 h c q}, \mu_{2 h c q}, \mu_{3 h c q}, \mu_{2 b c g b d}, \mu_{3 b c g b d}\right)\right| \rightarrow \infty$, where $|$.$| is an l^{2}(0, T)$ norm.

Now we will show that each of the conditions are satisfied :

1. From positivity and boundedness of solutions of the system (4.1) - (4.4) all the solutions of the system are bounded for each bounded control variable in $U$. Also, the right hand side of the system (4.1) - (4.4) satisfies Lipschitz condition with respect to state variables. Hence, using the positivity and boundedness condition and the existence of solution from Picard-Lindelof Theorem [30], we have satisfied condition 1.

2. $U$ is closed and convex by definition. Also, the system (4.1) - (4.4) is clearly linear with respect to controls such that coefficients are only state variables or functions dependent on time. Hence condition 2 is satisfied. 
3. Choosing $g\left(\mu_{1 h c q}, \mu_{2 h c q}, \mu_{3 h c q}, \mu_{2 b c g b d}, \mu_{3 b c g b d}\right)$

$=c\left(\mu_{1 h c q}^{2}+\mu_{2 h c q}^{2}+\mu_{3 h c q}^{2}+\mu_{2 b c g b d}^{2}+\mu_{3 b c g b d}^{2}\right)$ such that $c=\min \left\{A_{3}, A_{4}\right\}$, we can satisfy the condition 3 .

Hence there exists a control 5-tuple $\left(\mu_{1 h c q}, \mu_{2 h c q}, \mu_{3 h c q}, \mu_{2 b c g b d}, \mu_{3 b c g b d}\right) \in U$ that minimizes the cost function (4.4).

\subsection{Characterization of Optimal Controls}

We now obtain the necessary conditions for optimal control functions using the Pontryagin's Maximum Principle [27] and also obtain the characteristics of the optimal controls.

The Hamiltonian for this problem is given by

$$
H\left(S, I, V, U_{3}, U_{4}, \lambda\right)=L\left(S, I, V, U_{3}, U_{4}\right)+\lambda_{1} \frac{\mathrm{d} S}{\mathrm{~d} t}+\lambda_{2} \frac{\mathrm{d} I}{\mathrm{~d} t}+\lambda_{3} \frac{\mathrm{d} V}{\mathrm{~d} t}
$$

Here $\lambda=\left(\lambda_{1}, \lambda_{2}, \lambda_{3}\right)$ is called co-state vector or adjoint vector.

Now the Canonical equations that relate the state variables to the co-state variables are given by

$$
\begin{aligned}
\frac{\mathrm{d} \lambda_{1}}{\mathrm{~d} t} & =-\frac{\partial H}{\partial S} \\
\frac{\mathrm{d} \lambda_{2}}{\mathrm{~d} t} & =-\frac{\partial H}{\partial I} \\
\frac{\mathrm{d} \lambda_{3}}{\mathrm{~d} t} & =-\frac{\partial H}{\partial V}
\end{aligned}
$$

Substituting the Hamiltonian value gives the canonical system

$$
\begin{aligned}
& \frac{\mathrm{d} \lambda_{1}}{\mathrm{~d} t}=\lambda_{1}\left(\beta V+\mu+\mu_{1 h c q}\right)-\lambda_{2} \beta V \\
& \frac{\mathrm{d} \lambda_{2}}{\mathrm{~d} t}=1+\lambda_{2}\left(x+\left(\mu_{2 h c q}+\mu_{2 b c g b d}+\alpha_{1}+\mu\right)\right)-\lambda_{3} \alpha \\
& \frac{\mathrm{d} \lambda_{3}}{\mathrm{~d} t}=1+\lambda_{1} \beta S-\lambda_{2} \beta S+\lambda_{3}\left(y+\alpha_{2}+\mu_{3 b c g b d}+\mu_{3 h c q}+\mu_{1}\right)
\end{aligned}
$$

where $x=d_{1}+d_{2}+d-3+d_{4}+d_{5}+d_{6}$ and $y=b_{1}+b_{2}+b_{3}+b_{4}+b_{5}+b_{6}$ along with transversality conditions $\lambda_{1}(T)=0, \lambda_{2}(T)=0, \lambda_{3}(T)=0$.

Now, to obtain the optimal controls, we will use the Hamiltonian minimization condition $\frac{\partial H}{\partial u_{i}}=0$, at $\mu^{*}$. 
Differentiating the Hamiltonian and solving the equations, we obtain the optimal controls as

$$
\begin{aligned}
\mu_{1 h c q}^{*} & =\min \left\{\max \left\{\frac{\lambda_{1} S}{2 A_{3}}, 0\right\}, \mu_{1 h c q} \max \right\} \\
\mu_{2 h c q}^{*} & =\min \left\{\max \left\{\frac{\lambda_{2} I}{2 A_{3}}, 0\right\}, \mu_{2 h c q} \max \right\} \\
\mu_{3 h c q}^{*} & =\min \left\{\max \left\{\frac{\lambda_{3} V}{2 A_{3}}, 0\right\}, \mu_{3 h c q} \max \right\} \\
\mu_{2 b c g b d}^{*} & =\min \left\{\max \left\{\frac{\lambda_{2} I}{2 A_{4}}, 0\right\}, \mu_{2 b c g b d} \max \right\} \\
\mu_{3 b c g b d}^{*} & =\min \left\{\max \left\{\frac{\lambda_{3} V}{2 A_{4}}, 0\right\}, \mu_{3 b c g b d} \max \right\}
\end{aligned}
$$

\section{BIBLIOGRAPHY}

[1] https://arxiv.org/abs/2005.02261.

[2] https://www.dailymail.co.uk/sciencetech/article-8509655/bcg-does-protectagainst-covid-19-study-confirms.html.

[3] https://www.ndtv.com/india-news/health-ministry-okays-anti-malarial-drug-hcqfor-early-course-of-covid-19-2245829.

[4] https://www.newscientist.com/article/2242866-bcg-vaccine-helps-fightinfections-by-boosting-immune-cell-production/.

[5] https://www.tribuneindia.com/news/nation/hcq-vitamin-tablets-help-mumbaicops-beat-covid-19-96751.

[6] https://www.worldometers.info/coronavirus/.

[7] M Soledad Aronna, Roberto Guglielmi, and Lucas M Moschen, A model for covid-19 with isolation, quarantine and testing as control measures, arXiv preprint arXiv:2005.07661 (2020).

[8] Samia Arshad, Paul Kilgore, Zohra S Chaudhry, Gordon Jacobsen, Dee Dee Wang, Kylie Huitsing, Indira Brar, George J Alangaden, Mayur S Ramesh, John E McKinnon, et al., Treatment with hydroxychloroquine, azithromycin, 
and combination in patients hospitalized with covid-19, International journal of infectious diseases 97 (2020), 396-403.

[9] Martha K Berg, Qinggang Yu, Cristina E Salvador, Irene Melani, and Shinobu Kitayama, Mandated bacillus calmette-guérin (bcg) vaccination predicts flattened curves for the spread of covid-19, Medrxiv (2020).

[10] Xiaohua Chen, Binghong Zhao, Yueming Qu, Yurou Chen, Jie Xiong, Yong Feng, Dong Men, Qianchuan Huang, Ying Liu, Bo Yang, et al., Detectable serum sars-cov-2 viral load (rnaaemia) is closely associated with drastically elevated interleukin 6 (il-6) level in critically ill covid-19 patients, medRxiv (2020).

[11] Bishal Chhetri, Vijay M Bhagat, DKK Vamsi, VS Ananth, Bhanu Prakash, Roshan Mandale, Swapna Muthusamy, and Carani B Sanjeevi, Within-host mathematical modeling on crucial inflammatory mediators and drug interventions in covid19 identifies combination therapy to be most effective and optimal, Alexandria Engineering Journal (2020).

[12] Camila Covián, Angello Retamal-Díaz, Susan M Bueno, and Alexis M Kalergis, Could bcg vaccination induce protective trained immunity for sars-cov-2?, Frontiers in Immunology 11 (2020), 970.

[13] Ramses Djidjou-Demasse, Yannis Michalakis, Marc Choisy, Micea T Sofonea, and Samuel Alizon, Optimal covid-19 epidemic control until vaccine deployment, medRxiv (2020).

[14] _ Optimal covid-19 epidemic control until vaccine deployment, medRxiv (2020).

[15] Jeffrey W Eaton, Leigh F Johnson, Joshua A Salomon, Till Bärnighausen, Eran Bendavid, Anna Bershteyn, David E Bloom, Valentina Cambiano, Christophe Fraser, Jan AC Hontelez, et al., Hiv treatment as prevention: systematic comparison of mathematical models of the potential impact of antiretroviral therapy on hiv incidence in south africa, PLoS Med 9 (2012), no. 7, e1001245.

[16] Luis E Escobar, Alvaro Molina-Cruz, and Carolina Barillas-Mury, Bcg vaccine protection from severe coronavirus disease 2019 (covid-19), Proceedings of the National Academy of Sciences (2020).

[17] Wendell H Fleming and Raymond W Rishel, Deterministic and stochastic optimal control, vol. 1, Springer Science \& Business Media, 2012. 
[18] Winston Garira and Dephney Mathebula, Development and application of multiscale models of acute viral infections in intervention research, Mathematical Methods in the Applied Sciences 43 (2020), no. 6, 3280-3306.

[19] Evangelos J Giamarellos-Bourboulis, Maria Tsilika, Simone Moorlag, Nikolaos Antonakos, Antigone Kotsaki, Jorge Domínguez-Andrés, Evdoxia Kyriazopoulou, Theologia Gkavogianni, MariaEvangelia Adami, Georgia Damoraki, et al., Activate: randomized clinical trial of bcg vaccination against infection in the elderly, Cell 183 (2020), no. 2, 315323.

[20] Giulia Giordano, Franco Blanchini, Raffaele Bruno, Patrizio Colaneri, Alessandro Di Filippo, Angela Di Matteo, and Marta Colaneri, Modelling the covid-19 epidemic and implementation of population-wide interventions in italy, Nature Medicine (2020), 1-6.

[21] Marcus A Horwitz, Günter Harth, Barbara Jane Dillon, and Saša Masleša-Galić, Commonly administered bcg strains including an evolutionarily early strain and evolutionarily late strains of disparate genealogy induce comparable protective immunity against tuberculosis, Vaccine 27 (2009), no. 3, 441-445.

[22] Adam J Kucharski, Timothy W Russell, Charlie Diamond, Yang Liu, John Edmunds, Sebastian Funk, Rosalind M Eggo, Fiona Sun, Mark Jit, James D Munday, et al., Early dynamics of transmission and control of covid-19: a mathematical modelling study, The lancet infectious diseases (2020).

[23] Sunmi Lee, Gerardo Chowell, and Carlos Castillo-Chávez, Optimal control for pandemic influenza: the role of limited antiviral treatment and isolation, Journal of Theoretical Biology 265 (2010), no. 2, 136-150.

[24] Jenneke Leentjens, Matthijs Kox, Robin Stokman, Jelle Gerretsen, Dimitri A Diavatopoulos, Reinout van Crevel, Guus F Rimmelzwaan, Peter Pickkers, and Mihai G Netea, Bcg vaccination enhances the immunogenicity of subsequent influenza vaccination in healthy volunteers: a randomized, placebo-controlled pilot study, The Journal of infectious diseases 212 (2015), no. 12, 1930-1938.

[25] Chentong $\mathrm{Li}$, Jinhu $\mathrm{Xu}$, Jiawei Liu, and Yicang Zhou, The within-host viral kinetics of sars-cov-2, bioRxiv (2020).

[26] Xiaowei Li, Manman Geng, Yizhao Peng, Liesu Meng, and Shemin Lu, Molecular immune pathogenesis and diagnosis of covid-19, Journal of Pharmaceutical Analysis (2020). 
[27] Daniel Liberzon, Calculus of variations and optimal control theory: a concise introduction, Princeton University Press, 2011.

[28] Gustavo Barbosa Libotte, Fran Sérgio Lobato, Gustavo Mendes Platt, and Antônio J Silva Neto, Determination of an optimal control strategy for vaccine administration in covid-19 pandemic treatment, Computer methods and programs in biomedicine 196 (2020), 105664.

[29] Yingxia Liu, Yang Yang, Cong Zhang, Fengming Huang, Fuxiang Wang, Jing Yuan, Zhaoqin Wang, Jinxiu Li, Jianming Li, Cheng Feng, et al., Clinical and biochemical indexes from 2019-ncov infected patients linked to viral loads and lung injury, Science China Life Sciences 63 (2020), no. 3, 364-374.

[30] Evgeny Makarov and Bas Spitters, The picard algorithm for ordinary differential equations in coq, International Conference on Interactive Theorem Proving, Springer, 2013, pp. 463-468.

[31] E Mohamed, R Mostafa, and B Elhabib, Optimal control of an sir model with delay in state and control variables, ISRN Biomathematics (2013).

[32] Prasanta Raghab Mohapatra, Baijayantimala Mishra, and Bijayini Behera, Bcg vaccination induced protection from covid-19, Indian Journal of Tuberculosis (2020).

[33] Simone JCFM Moorlag, Rosanne C van Deuren, Cornelis H van Werkhoven, Martin Jaeger, Priya Debisarun, Esther Taks, Vera P Mourits, Valerie ACM Koeken, L Charlotte J de Bree, Thijs Ten Doesschate, et al., Safety and covid19 symptoms in individuals recently vaccinated with bcg: a retrospective cohort study, Cell Reports Medicine (2020), 100073.

[34] Nicole Ritz, Willem A Hanekom, Roy Robins-Browne, Warwick J Britton, and Nigel Curtis, Influence of bcg vaccine strain on the immune response and protection against tuberculosis, FEMS microbiology reviews 32 (2008), no. 5, $821-841$.

[35] Eugenia Quiros Roldan, Giorgio Biasiotto, Paola Magro, and Isabella Zanella, The possible mechanisms of action of 4-aminoquinolines (chloroquine/hydroxychloroquine) against sars-cov-2 infection (covid-19): A role for iron homeostasis?, Pharmacological research (2020), 104904. 
[36] Piu Samui, Jayanta Mondal, and Subhas Khajanchi, A mathematical model for covid-19 transmission dynamics with a case study of india, Chaos, Solitons \& Fractals 140 (2020), 110173.

[37] Esteban Abelardo Hernandez Vargas and Jorge X Velasco-Hernandez, In-host modelling of covid-19 kinetics in humans, medRxiv (2020).

[38] Changjin Xu and Peiluan Li, Oscillations for a delayed predator-prey model with hassell-varley-type functional response, Comptes rendus biologies 338 (2015), no. 4, 227-240.

[39] Changjin $\mathrm{Xu}$, Peiluan $\mathrm{Li}$, and Maoxin Liao, Periodic property and asymptotic behavior for a discrete ratio-dependent food-chain system with delays, Discrete Dynamics in Nature and Society 2020 (2020).

[40] Changjin Xu, Peiluan Li, Maoxin Liao, and Shuai Yuan, Bifurcation analysis for a fractional-order chemotherapy model with two different delays, Mathematical Methods in the Applied Sciences 43 (2020), no. 3, 1053-1083.

[41] Changjin $\mathrm{Xu}$, Maoxin Liao, and Peiluan Li, Bifurcation of a fractional-order delayed malware propagation model in social networks, Discrete Dynamics in Nature and Society 2019 (2019).

[42] Chayu Yang and Jin Wang, A mathematical model for the novel coronavirus epidemic in wuhan, china, Mathematical Biosciences and Engineering 17 (2020), no. 3, 2708-2724.

[43] Sherif R Zaki and Cynthia S Goldsmith, Sars coronavirus infection: pathology and pathogenesis of an emerging virus disease, Coronaviruses with Special Emphasis on First Insights Concerning SARS, Springer, 2005, pp. 87-99. 